\title{
Research Note on Decision Lists
}

RON KOHAVI

RONNYK@CS.STANFORD.EDU

SCOTT BENSON

SBENSON@CS.STANFORD.EDU

Computer Science Dept., Stanford University, Stanford, CA 94305

Editor: David Haussler

Abstract. In his article "Learning Decision Lists," Rivest proves that ( $k$-DNF $\cup k$-CNF) is a proper subset of $k$-DL. The proof is based on the following incorrect claim:

$\ldots$ if a function $f$ has a prime implicant of size $t$, then $f$ has no $k$-DNF representation if $k<t$.

In this note, we show a counterexample to the claim and then prove a stronger theorem, from which Rivest's theorem follows as a corollary.

\section{A counterexample}

In the article "Learning Decision Lists" (Rivest, 1987) Rivest proves that ( $k$-DNF $\cup k$-CNF) is a proper subset of $k$-DL. The proof is based on the following incorrect claim:

.. if a function $f$ has a prime implicant of size $t$, then $f$ has no $k$-DNF representation if $k<t$.

The following counterexample shows that it is possible for a function $f$ with a prime implicant of size four to have a 3-DNF representation. The function $f$ shown below is in 3-DNF, yet the term $w \bar{x} y \bar{z}$ is a prime implicant of the function.

$$
f(v, w, x, y, z)=v w \bar{x} \vee \bar{v} y \bar{z}
$$

Figure 1 shows the function using a Karnaugh map of five variables with the prime implicant containing four literals shaded. (For a description of Karnaugh maps, see, for example, Kohavi (1978) or Friedman (1986), although readers not familiar with them may easily check that the given term is indeed a prime implicant.)

\section{The expressive power of decision lists}

Let $n$ be the number of variables in our language.

Definition 1 (Prime implicant). A prime implicant for a function $f$ is a product term $\alpha$ that implies $f$, but that does not imply $f$ if any literal in $\alpha$ is deleted. 


\begin{tabular}{|c|c|c|c|c|c|c|c|c|}
\hline $\begin{array}{c}\text { vyyz } \\
\text { Vw }\end{array}$ & 000 & 001 & 011 & 010 & 110 & 111 & 101 & 100 \\
\hline 00 & 0 & 0 & 0 & 1 & 1 & 0 & 0 & 0 \\
\hline 01 & 0 & 0 & 0 & & 1 & 0 & 0 & 0 \\
\hline 11 & 1 & 1 & 1 & 0 & 0 & 0 & 0 & 0 \\
\hline 10 & 0 & 0 & 0 & 0 & 0 & 0 & 0 & 0 \\
\hline
\end{tabular}

Figure 1. A Karnaugh map that refutes the claim.

Definition 2 (Essential prime implicant). An essential prime implicant $\alpha$ of $f$ is a prime implicant such that there exists an $x \in\{0,1\}^{n}$ with $\alpha(x)=1$, yet for no prime implicant $\beta \neq \alpha$ does $\beta(x)=1$.

Lemma 1. If a function fhas an essential prime implicant of size $t$, then fhas no $k-D N F(n)$ representation if $k<t$.

Proof: The essential prime implicant must appear in any $\operatorname{DNF}(n)$ representation that uses only prime implicants. Any $k-\mathrm{DNF}(n)$ representation has an equivalent $k-\mathrm{DNF}(n)$ representation using only prime implicants; therefore, there cannot exist a $k-\operatorname{DNF}(n)$ representation of $f$ with $k<t$.

Note that this lemma only defines a sufficient condition for not having a $k$ - DNF $(n)$ representation. There are functions that have no essential prime implicants at all.

Lemma 2. A prime implicant $\alpha$ of size $n$ is an essential prime implicant.

Proof: Let $x \in\{0,1\}^{n}$ be the unique vector such that $\alpha(x)=1$. If there exists a prime implicant $\beta \neq \alpha$ for which $\beta(x)=1$, then $\alpha$ and $\beta$ cannot disagree on any literal (or else $\beta(x) \neq 1)$. Since all variables appear in $\alpha$, the prime implicant $\beta$ must contain only a subset of the literals in $\alpha$, contradicting the fact that $\alpha$ is a prime implicant.

Theorem 3. For $1<k<n$ and $n>2$, there are functions representable in $k-D L(n)$ but not in $(j-C N F(n) \cup j-D N F(n))$ for any $j<n$.

Proof: We prove a stronger result, namely, that 2-DL( $n$ ) contains functions not representable in $(j-\operatorname{CNF}(n) \cup j-\operatorname{DNF}(n))$ for any $j<n$, and $n>2$.

Let $f$ be the function represented by the following 2-DL $(n)$ :

$\left(\overline{x_{1}} \overline{x_{2}}, 0\right),\left(\overline{x_{1}} \overline{x_{3}}, 0\right), \ldots,\left(\overline{x_{1}} \overline{x_{\mathrm{n}}}, 0\right),\left(\overline{x_{1}}, 1\right),\left(x_{1} \overline{x_{2}}, 1\right),\left(x_{1} \overline{x_{3}}, 1\right), \ldots,\left(x_{1} \overline{x_{n}}, 1\right),($ true, 0$)$ 


\begin{tabular}{|c|c|c|c|c|c|c|c|c|}
\hline $\begin{array}{l}x_{1} x_{2} x_{3} \\
x_{4} x_{5}\end{array}$ & 000 & 001 & 011 & 010 & 110 & 111 & 101 & 100 \\
\hline 00 & 0 & 0 & 0 & 0 & 1 & 1 & 1 & 1 \\
\hline 01 & 0 & 0 & 0 & 0 & 1 & 1 & 1 & 1 \\
\hline 11 & 0 & 0 & 1 & 0 & 1 & 0 & 1 & 1 \\
\hline 10 & 0 & 0 & 0 & 0 & 1 & 1 & 1 & 1 \\
\hline
\end{tabular}

Figure 2. A Karnaugh map showing the funciton in 2-DL( $(n)$ for $n=5$.

Note that the last term could be replaced by $\left(x_{1}, 0\right)$, but the definition of a decision list requires the last term to contain the constant function true. Figure 2 shows a Karnaugh map of the function for $n=5$.

Let $\alpha$ be the term $\overline{x_{1}} x_{2} x_{3} \ldots x_{n}$ and let $\alpha^{\prime}$ be a term derived from $\alpha$ with one literal $l_{i}$ deleted. $\alpha^{\prime}$ implies $\alpha^{\prime} l_{i}$, but for any $\vec{x} \in\{0,1\}^{n}$ such that $\alpha \tau_{i}$ is true, $f(\vec{x})$ is 0 , and thus $\alpha$ is a prime implicant of $f$. By lemma $2, \alpha$ is an essential prime implicant, and by lemma 1 , $f$ has no $j$-DNF $(n)$ representation for $j<n$.

Similarly, the term $x_{1} x_{2} x_{3} \ldots x_{n}$ is an essential prime implicant of $\bar{f}$, and thus the function $\bar{f}$ cannot be represented in $j-\operatorname{DNF}(n)$ for $j<n$. Since the complement of every $j$-CNF $(n)$ formula is a $j-\operatorname{DNF}(n)$ formula, there is no $j-\operatorname{DNF}(n)$ representation for $f$, and hence $f$ cannot be represented in $j-\operatorname{DNF}(n) \cup j-\operatorname{CNF}(n)$ for $j<n$.

Corollary 4 (Rivest). For $0<k<n$ and $n>2,(k-C N F(n) \cup k$-DNF(n)) is a proper subset of $k-D L(n)$.

Proof: The original article (Rivest, 1987) correctly proved that any $k-\mathrm{CNF}(n)$ formula and any $k-\mathrm{DNF}(n)$ formula can be written in $k-\mathrm{DL}(n)$. By theorem 3 , there are functions in $k$-DL $(n)$ not in $(k-\mathrm{CNF}(n) \cup k$ - DNF $(n))$ for $k>1$, so only the case $k=1$ remains to be proved.

If $k=1$, then the following decision list from 1-DL( $n)$ represents a function $f$ that is not in $1-\operatorname{CNF}(n) \cup 1-\mathrm{DNF}(n)$ :

$$
\left.\left(x_{1}, 0\right),\left(x_{2}, 1\right),\left(x_{3}, 1\right), \text { (true, } 0\right)
$$

The only prime implicants of the function $f$ are $\overline{x_{1}} x_{2}$ and $\overline{x_{1}} x_{3}$. Both are essential, so $f$ does not have a 1-DNF $(n)$ representation. Similarly, the function $\bar{f}$ has $x_{1}$ and $\overline{x_{2}} \overline{x_{3}}$ as the only prime implicants and again both are essential, so $f$ does not have a 1-DNF $(n) \cup$ $1-\mathrm{CNF}(n)$ representation. 


\section{Acknowledgments}

We thank the anonymous referee for comments on making the proof shorter. We thank Nils Nilsson, Ron Rivest, and George John for their comments on a previous version of this article, and Shai Halevi for coming up with the counterexample, which is smaller than our original one.

\section{References}

Friedman, Arthur D. (1986). Fundamentals of logic design and switching. Computer Science Press. Kohavi, Zvi. (1978). Switching and finite automata theory, 2nd edition. New York: McGraw-Hill. Rivest, Ronald L. (1987). Learning decision lists. Machine Learning, 2, 229\#246.

Received July 30, 1992

Accepted October 7, 1992

Final Manuscript February 18, 1993 